\title{
A study of benzotriazole and mercaptobenzothiazole as inhibiting additives to mineral oil
}

\author{
S.M. Reshetnikov, ${ }^{1,2}$ iD ${ }^{\text {I.N. Shabanova, }}{ }^{1}$ E.A. Naimushina, ${ }^{1,3}$ \\ N.S. Terebova ${ }^{1}$ and A.V. Kholzakov ${ }^{1}$ \\ ${ }^{1}$ Udmurt Federal Research Center of the Ural Branch of the Russian Academy of Sciences, \\ 34 T. Baramzina street, 426067 Izhevsk, Russian Federation \\ ${ }^{2}$ Udmurt State University, 1 Universitetskaya street, 426034 Izhevsk, Russian Federation \\ ${ }^{3}$ Kalashnikov Izhevsk State Technical University, 7 Studencheskaya street, 426069 Izhevsk, \\ Russian Federation \\ *E-mail: xps@udman.ru
}

\begin{abstract}
The principal feasibility of developing protective mineral oil formulations using inhibitive additives such as 1,2,3-benzotriazole (BTA) и mercaptobenzothiazole (MBTA), well-known and available corrosion inhibitors in aqueous media, has been shown. The protective effect of these formulations was studied using a Monicor corrosimeter in a corrosive medium that simulates the condensed moisture in industrial cities. The protective effect of mineral oil formulations containing 1-5 mass\% of BTA and MBTA is only exhibited in full if the layer of the formulation applied onto non-alloy steel is heated to $50-150^{\circ} \mathrm{C}$. XPS studies have shown that chemisorption bonds of iron atoms with nitrogen atoms in the BTA molecule and nitrogen and sulfur atoms in the MBTA molecule are formed upon such heating (thermochemical activation). It is due to this interaction of inhibitor atoms with iron atoms on the surface of steel, which is initiated by thermochemical activation, that a dense protective layer is formed. Having measured the masses of the samples prior to and after application of the layer, we calculated the coating thickness. It proved to be $c a .100 \mathrm{~nm}$, which would allow one to skip the depreservation stage after inter-operation protection of fine-mechanic equipment if the formulations studied are used.
\end{abstract}

Keywords: X-ray photoelectron spectroscopy, corrosimeter, protective anticorrosion layer, corrosion inhibitors, mercaptobenzothiazole (MBTA), benzotriazole (BTA).

Received: May 21, 2021. Published: July 14, 2021

doi: $\underline{10.17675 / 2305-6894-2021-10-3-6}$

\section{Introduction}

Significant attention has been paid lately to exploration of the properties of azole-based corrosion inhibitors [1-3]. Studies of 1,2,3-benzotriazole (BTA) and mercaptobenzothiazole (MBTA) have been the most detailed and comprehensive. These commercially available inhibitors have shown their efficiency in both acid and neutral corrosive media. 
Data on the XPS spectra of these inhibitors on iron surface are available. It was observed, in particular, that iron atoms formed bonds with nitrogen atoms of triazoles, which may be an indication of chemisorption of the inhibitors [4]. However, studies of iron samples pretreated with laser radiation under various conditions [5] have shown that fairly dense and thick layers of adsorbed oxygen, which may be considered as production-related surface iron oxides, may worsen BTA adsorption conditions and reduce its inhibiting efficiency.

It should be noted that adding BTA to a paint-based coating [6] yielded a fairly large enhancement of the BTA inhibitory efficiency. It may be related to the fact that in this method of inhibition where the surface of iron does not contact water and a continuous oxide (hydroxide) layer is not formed on this surface, conditions for adsorption of BTA particles become more favorable. In view of this, it seemed possible to use azole-class inhibitors, in particular, BTA and MBTA, as inhibiting additives to petroleum oils in order to enhance their protective properties.

The purpose of this study was to explore the protective properties of oil formulations using BTA and MBTA as the anticorrosion agents and the interatomic interaction of the molecules of these additives with the surface of the metal being protected.

\section{Experimental}

Vacuum oil VM-1S (Technical specifications (TU) 38.1011187-88) was chosen as the oil base. Unlike other petroleum oils, this oil consists of pure hydrocarbons without any volatile components or products of chemical conversion of hydrocarbons. It should be stressed that this oil was selected as a model of an oil medium. The inhibitors under study were added to the oil in an amount of 1 to $5 \mathrm{wt} \%$. Addition of inhibitors BTA and MBTA within the studied range of 1-5 wt \% does not change the oil viscosity, i.e., it may be assumed that the thickness of the oil layer remains the same as that of the oil without additives.

Steel samples with dimensions of $20 \times 20 \times 2 \mathrm{~mm}$ were sandpapered (sandpaper R600) and covered with two layers of Zapon lacquer. A working area of $400 \mathrm{~mm}^{2}$ was left uncoated. The samples mounted in the corrosimeter probe were then immersed for 5-7 min into the protective (preservative) formulations under study. If a coated sample was heated in a muffle furnace, the non-working surfaces were insulated with the lacquer after cooling the sample. A segment $400 \mathrm{~mm}^{2}$ in area was used as the working surface that was exposed in the corrosimeter cell. A solution containing $30 \mathrm{mg} / \mathrm{L} \mathrm{NaCl}$ and $70 \mathrm{mg} / \mathrm{L} \mathrm{Na} \mathrm{SO}_{4}$, which simulates the moisture condensate of industrial cities as per national standard GOST 9.50282 , item 5 , was used as the corrosive medium.

The corrosion rate of the samples was measured using a Monicor corrosimeter. In studies of protective coatings, including those based on mineral oil, the results obtained using a corrosimeter can be estimated in the corrosion rate units $\left(\mathrm{mm} / \mathrm{year}, \mathrm{g} / \mathrm{m}^{2} \cdot\right.$ hour $)$ in only formally. This is due to the fact that there are almost no Tafel slopes, especially on cathodic curves, in the presence of mineral oil layers [7]. Therefore, the corrosion rate of original 
samples without any coating was taken as a unity, and all the other data were referred to this value.

The following notations were used in the text and in the table for labeling samples and the treatment modes:

00 -steel 08: cleaned, degreased, uncoated. Such samples were tested in the background solution.

0 - same sample but coated with an oil film obtained by the following procedure: the sample is immersed into oil at room temperature and kept there for $10 \mathrm{~min}$; the oil flows down under gravity, and the sample is kept for $10 \mathrm{~min}$ in atmospheric air;

1 - steel sample coated with an oil layer containing 1 mass $\%$ of the inhibitor;

3 - steel sample coated with an oil layer containing 3 mass $\%$ of the inhibitor;

5 - steel sample coated with an oil layer containing 5 mass $\%$ of the inhibitor.

The increase in the sample mass was used to calculate the thickness of the oil layer with consideration for the oil density and under the assumption that the oil layer is uniformly distributed over the sample surface; it amounted to $0.1 \mu \mathrm{m}$ or $100 \mathrm{~nm}$.

XPS studies were conducted using an XPS magnetic spectrometer with $10^{-4}$ resolution

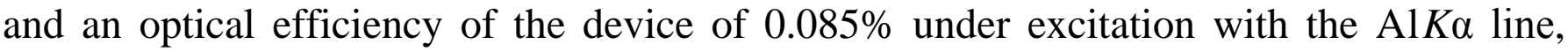
$1486.5 \mathrm{eV}$ [8].

\section{Results and Discussion}

As noted above, addition of 1-5 mass \% BTA and MBTA inhibitors to the oil does not change the oil viscosity, i.e., it may be assumed that the thickness of the oil layer is the same as in the case where the oil is used without additives.

The results of testing in the corrosive medium used with a Monicor corrosimeter after application of coating based on oil formulations are displayed in the table.

The samples mounted in the corrosimeter probe were immersed into the solution. The corrosion rate $\left(V_{\text {rel }}\right)$ was measured for two hours. After this time interval elapsed, the corrosion rate attained a constant value, which is shown in Table 1. The table also displays the corrosion rate reduction coefficients $(\gamma)$ of the samples that have a protective oil layer with the inhibitors. The corrosion rate reduction coefficient was calculated using the formula $\gamma=V_{0} / V_{\mathrm{i}}$, where $V_{0}$ is the corrosion rate of the sample without a protective layer and $V_{\mathrm{i}}$ is the corrosion rate of the sample coated with a protective layer.

One can see that the oil without inhibitors shows weak protective properties at room temperature, as it was repeatedly reported in publications [7]. Addition of BTA and MBTA inhibitors significantly reduces the corrosion rate of the samples.

We have shown previously [9] that heating of samples coated with a protective layer that contains an oil-soluble inhibitor enhances the protective properties significantly. This is due to the activation of inhibitor particles and facilitation of the formation of adsorption bonds on heating. We call this thermal treatment as thermochemical activation. 
Samples coated with an inhibited oil layer were heated in a muffle furnace. The samples were maintained in the heating zone for $15 \mathrm{~min}$. Uncoated samples and samples with the oil coating containing no inhibitors were heated for comparison.

Table 1 shows that heating of samples both without an oil layer and with an oil layer containing no inhibitors reduces the measured corrosion rate to some extent only.

Table 1. Corrosion rates of the sample coated with a protective oil layer with and without inhibitors and the corrosion rate reduction coefficients.

Conventional (relative) corrosion rate $\left(V_{\text {rel }}\right)$ and the corrosion rate reduction coefficient $(\gamma)$

\begin{tabular}{ccccccccccc}
\hline \multirow{2}{*}{ Sample } & Inhibitor & \multicolumn{8}{c}{ Thermochemical activation } \\
\cline { 3 - 11 } & & \multicolumn{2}{c}{$\mathbf{2 0}^{\circ} \mathbf{C}$} & \multicolumn{2}{c}{$\mathbf{5 0}^{\circ} \mathbf{C}$} & \multicolumn{2}{c}{$\mathbf{1 0 0}^{\circ} \mathbf{C}$} & \multicolumn{2}{c}{$\mathbf{1 5 0}^{\circ} \mathbf{C}$} \\
\cline { 3 - 11 } & & $\boldsymbol{V}_{\text {rel }}$ & $\gamma$ & $\boldsymbol{V}_{\text {rel }}$ & $\gamma$ & $\boldsymbol{V}_{\text {rel }}$ & $\gamma$ & $\boldsymbol{V}_{\text {rel }}$ & $\gamma$ \\
\hline 00 & none & 1 & 1 & 0.98 & 1.02 & 0.91 & 1.10 & 0.95 & 1.05 \\
\hline 0 & oil & 0.92 & 1.09 & 0.89 & 1.12 & 0.90 & 1.11 & 0.90 & 1.11 \\
\hline \multirow{2}{*}{1} & BTA & 0.68 & 1.47 & 0.28 & 3.57 & 0.1 & 10.00 & 0.12 & 8.33 \\
& MBTA & 0.63 & 1.59 & 0.23 & 4.35 & 0.08 & 12.50 & 0.04 & 25.00 \\
\hline \multirow{2}{*}{3} & BTA & 0.61 & 1.64 & 0.22 & 4.55 & 0.08 & 12.50 & 0.08 & 12.50 \\
& MBTA & 0.59 & 1.89 & 0.20 & 5.00 & 0.06 & 16.67 & 0.08 & 12.50 \\
\hline \multirow{2}{*}{5} & BTA & 0.60 & 1.67 & 0.22 & 4.55 & 0.08 & 12.50 & 0.09 & 11.11 \\
& MBTA & 0.58 & 1.72 & 0.20 & 5.00 & 0.06 & 16.67 & 0.03 & 33.33 \\
\hline
\end{tabular}

00 - uncoated steel; 0 - steel coated with the VM-1S oil; 1 - steel coated with a $1 \%$ solution of inhibitors in oil; 3 - steel coated with a $3 \%$ solution of inhibitors in oil; 5 - steel coated with a $5 \%$ solution of inhibitors in oil.

It should be noted at the same time that heating to 50 or $100^{\circ} \mathrm{C}$ significantly enhances the efficiency of the inhibitors. However, if the BTA inhibitor is heated to $150^{\circ} \mathrm{C}$, its efficiency decreases somewhat. It may not be ruled out that this phenomenon may be due to the sublimation of BTA particles. It should be noted that heating the samples coated with oil layer in the presence of BTA or MBTA reduces the corrosion rate of the samples most efficiently upon thermochemical activation at $100^{\circ} \mathrm{C}$. It is also noteworthy that increasing the concentration of the inhibitors from 3 to 5 mass $\%$ affects the protective efficiency of the layer formed insignificantly. In view of this, it may be concluded that the optimal concentration of inhibitors under the conditions of our experiment is 3 mass $\%$ for thermochemical activation at a temperature not exceeding $100^{\circ} \mathrm{C}$. 
According to [7], the commercially available protective lubricants based on petroleum oils may contain a complex of several inhibitors with a total concentration of 25 mass $\%$ in the oil base.

In relation to this, certain advantages of the tested formulation may be noted as it contains rather a low concentration of additives.

To examine the interatomic interaction of the molecules of additives with the surface of the metal being protected, standard powders of BTA and MBTA inhibitors deposited on iron surface were studied by XPS.

The position of the maximum in the C1s spectra of BTA and MBTA corresponds to the $\mathrm{C}-\mathrm{H}$ bond in hydrocarbons $(285 \mathrm{eV})$; no carbide was found.

The N1s XPS spectrum of BTA (Figure 1, spectrum 1) without heating exhibits an intense maximum with a binding energy of $400.6 \mathrm{eV}$. After heating to $100^{\circ} \mathrm{C}$ (Figure 1, spectrum 2), the second component appears in the spectrum; its energy equal to $398.9 \mathrm{eV}$ is characteristic of the $\mathrm{N}-\mathrm{Fe}$ bond. The $400.6 \mathrm{eV}$ component that corresponds to the $\mathrm{N}-\mathrm{Fe}-\mathrm{O}$ bond persists. Unlike BTA, the N1s spectrum of MBTA contains two components already at room temperature: $\mathrm{N}-\mathrm{Fe}$ and $\mathrm{N}-\mathrm{Fe}-\mathrm{O}$ (Figure 1, spectrum 3). After heating to $100^{\circ} \mathrm{C}$ (Figure 1, spectrum 4), a relative growth of the $\mathrm{N}-\mathrm{Fe}$ component $(398.9 \mathrm{eV})$ is observed.

Figure 1 displays the N1s spectra of BTA and MBTA adsorbed on iron surface.

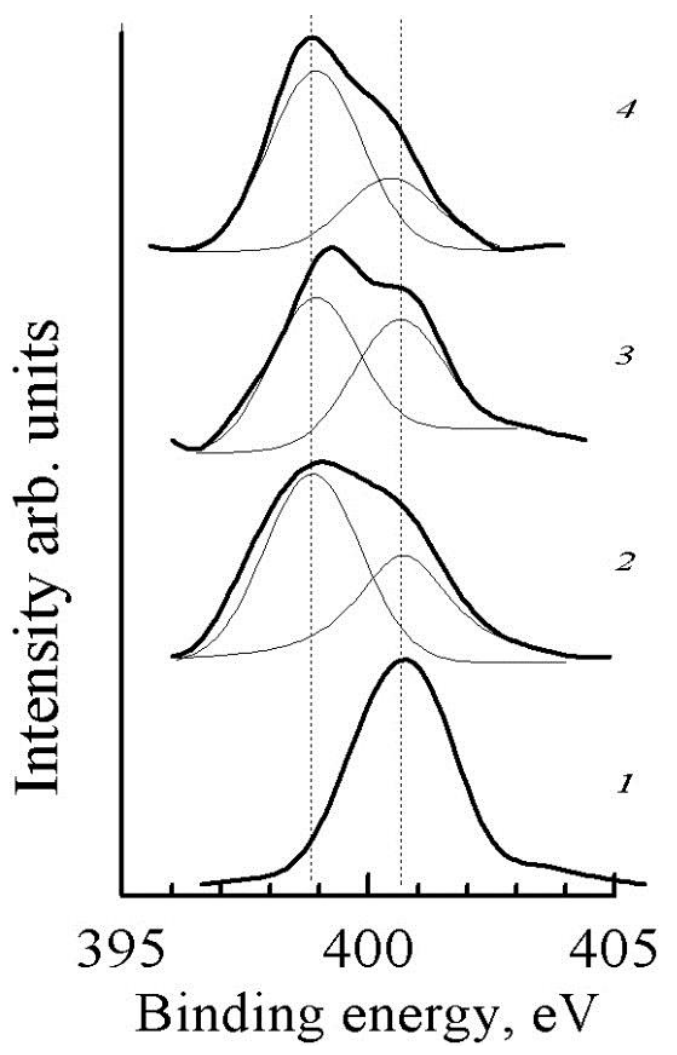

$100^{\circ} \mathrm{C}$ (spectrum 2); MBTA without heating (spectrum 3 ) and after heating to $100^{\circ} \mathrm{C}$ (spectrum 4).

Figure 1. XPS N1s spectra on iron surface: BTA without heating (spectrum 1) and after heating to 


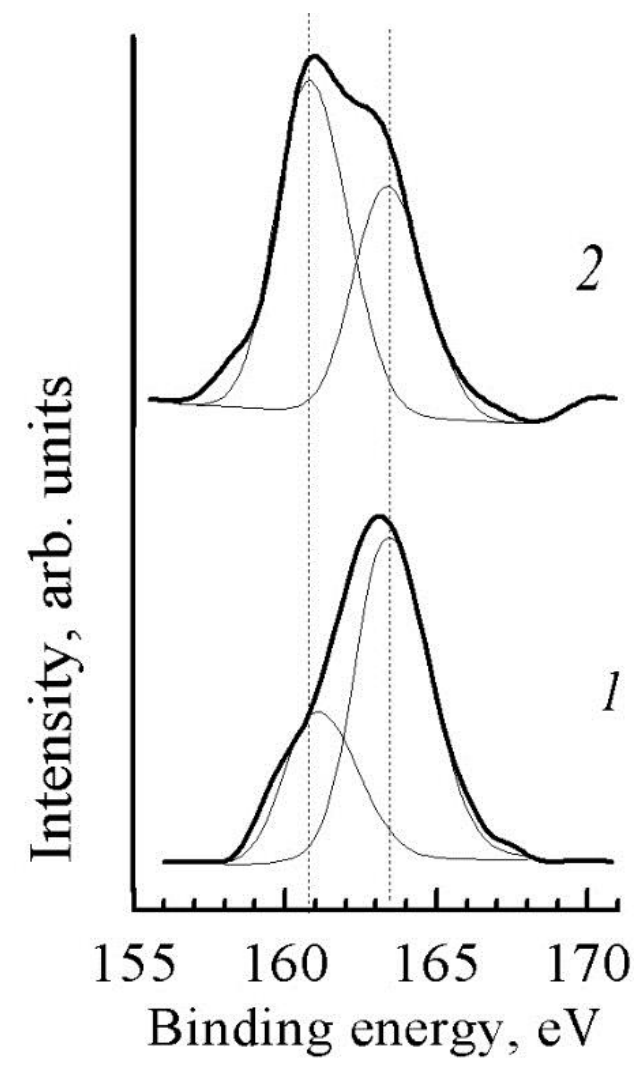

Figure 2. XPS S2p spectra of MBTA on iron surface: without heating (spectrum 1) and after heating to $100^{\circ} \mathrm{C}$.

Figure 2 shows the S2p spectra of MBTA on iron surface. The XPS S2p spectrum of the original MBTA powder without heating consists of two components and exhibits an intense maximum at $163.4 \mathrm{eV}$ corresponding to the $\mathrm{S}-\mathrm{Fe}-\mathrm{O}$ bond, and a maximum in the region of $160.8 \mathrm{eV}$ characteristic of the $\mathrm{S}-\mathrm{Fe}$ bond (Figure 2, spectrum 1).

After heating to $100^{\circ} \mathrm{C}$, the $\mathrm{S}-\mathrm{Fe}$ component in the region of $160.8 \mathrm{eV}$ increases (Figure 2, spectrum 2).

The Fe2p spectra of BTA and MBTA (Figure 3, spectra 1 and 3) on steel without heating contain, in addition to the region characteristic of $\mathrm{Fe}-\mathrm{O}_{x}$ oxides, a component that corresponds to pure iron $(707 \mathrm{eV})$. This is an indication that the surface area contains, aside from oxides, also areas of unoxidized iron. After heating to $100^{\circ} \mathrm{C}$, a component grows in the $\mathrm{Fe} 2 \mathrm{p}$ spectrum that has a binding energy of $707 \mathrm{eV}$ (Figure 3, spectra 2 and 4) and is characteristic of both pure iron and the covalent (donor-acceptor) bond of iron with nitrogen, $\mathrm{Fe}-\mathrm{N}$ (BTA), or with sulfur and nitrogen, Fe-S-N (MBTA). The formation of covalent bonds of iron with nitrogen and sulfur upon heating is confirmed by the emergence (BTA) or growth (MBTA) of components with smaller binding energies in the N1s and S2p spectra.

Figure 3 displays the Fe2p spectra of BTA and MBTA adsorbed on iron surface. 


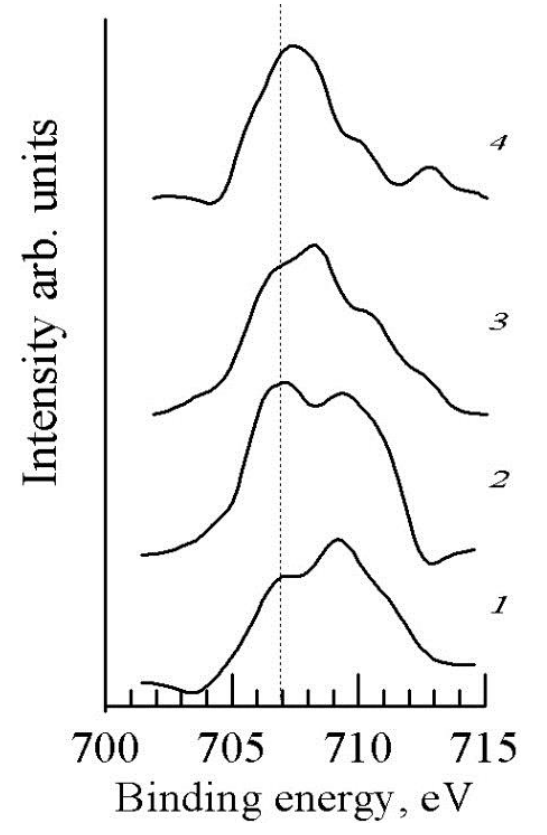

Figure 3. XPS Fe2p spectra on iron surface: BTA without heating (spectrum 1) and after heating to $100^{\circ} \mathrm{C}$ (spectrum 2); MBTA without heating (spectrum 3 ) and after heating to $100^{\circ} \mathrm{C}$ (spectrum 4);

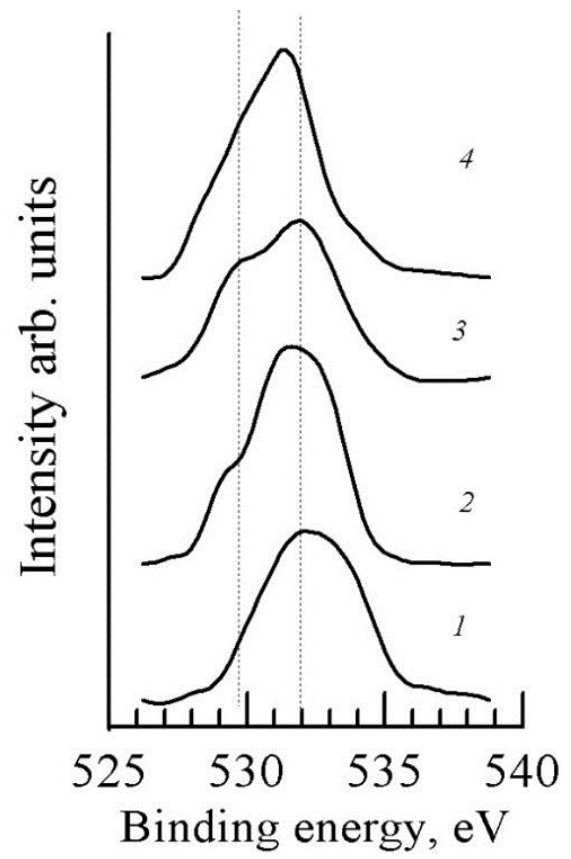

Figure 4. XPS O1s spectra on iron surface: BTA without heating (spectrum 1) and after heating to $100^{\circ} \mathrm{C}$ (spectrum 2); MBTA without heating (spectrum 3) and after heating to $100^{\circ} \mathrm{C}$ (spectrum 4);

In the absence of heating, the maximum in the O1s spectrum of BTA (Figure 4, spectrum 1) corresponds to adsorbed oxygen, while the O1s spectrum of MBTA (Figure 4, spectrum 3) contains two components from iron oxide $(530.0 \mathrm{eV}-\mathrm{Fe}-\mathrm{O})$ and adsorbed oxygen $\left(532.0 \mathrm{eV}-\mathrm{O}_{\mathrm{ads}}\right.$ ). After heating to $100^{\circ} \mathrm{C}$ (Figure 4, spectra 2 and 3), the contribution of adsorbed oxygen decreases, whereas the component characteristic of the $\mathrm{Fe}-\mathrm{O}$ bond $(530.0 \mathrm{eV})$ becomes more pronounced.

Comparison of the spectra obtained for steel surfaces with deposited BTA and MBTA powders indicates that the interaction between the inhibitors and iron is of chemisorption nature in both cases but is more pronounced for MBTA. The chemical interaction of MBTA with iron occurs due to the formation of covalent (donor-acceptor) bonds of iron atoms with nitrogen and sulfur atoms, while in BTA the covalent bond with the surface of iron is only due to nitrogen atoms.

Thus, the results obtained allow us to conclude that addition of corrosion inhibitors BTA and MBTA known as inhibitors for aqueous media to the model oil medium makes it possible to develop mineral oil formulations with a thin, down to $100 \mathrm{~nm}$, surface layer owing to which depreservation of products after inter-operation storage periods becomes unnecessary. 


\section{Conclusions}

The protective effect of a mineral oil-based formulation consisting of a vacuum oil with BTA и MBTA inhibitors added in an amount of 1 to 5 mass $\%$ has been studied.

The protective effect of this mineral oil formulation has been shown to increase after heating samples coated with this oil formulation to $50-150^{\circ} \mathrm{C}$.

Corrosion tests show that if the concentration of BTA and MBTA in the model medium is within 1-5 mass \%, the corrosion rate of the samples may be reduced by $80-95 \%$ after thermochemical activation at $50-100^{\circ} \mathrm{C}$.

The XPS spectra of the adsorption layers on the surface of non-alloy steel samples have been studied. It has been shown that an efficient protective layer is formed upon adsorption of a mineral oil formulation containing BTA or MBTA due to the formation of covalent (donor-acceptor) bonds of iron atoms with nitrogen atoms in the case of BTA-based mineral oil protective formulations and with nitrogen and sulfur atoms in the case of MBTA-based mineral oil protective formulations.

The principal feasibility of developing protective formulations based on mineral oil using available corrosion inhibitors well-known for aqueous media as inhibiting additives has been demonstrated.

\section{Acknowledgements}

The work was performed as part of a government contract (state registration No. 21030100002-0). The studies were carried out using the equipment provided by the shared "Center for physical and physicochemical methods of analysis, exploration of the properties and characteristics of surface, nanostructures, materials, and products" of the Udmurt Federal Research Center, Ural Branch of the Russian Academy of Sciences supported by the RF Ministry of Education and Science as part of the Special Federal Program "Research and Development in the Priority Areas of the Development of the Scientific and Technological Complex of Russia for the Years 2014-2020" (Unique project identifier: RFMEFI62119X0035).

\section{References}

1. Yu.I. Kuznetsov, Triazoles as a class of multifunctional corrosion inhibitors. Review. Part III. 1,2,3-Benzotriazole and its derivatives. Aluminum alloys, Int. J. Corros. Scale Inhib., 2020, 9, no. 4, 1142-1168. doi: 10.17675/2305-6894-2020-9-4-1

2. Ya.G. Avdeev and Yu.I. Kuznetsov, Inhibitory protection of steels in acid solutions under high-temperature corrosion conditions. A review. Part 3, Int. J. Corros. Scale Inhib., 2020, 9, no. 4, 1194-1236. doi: 10.17675/2305-6894-2020-9-4-3

3. Ya.G. Avdeev and Yu.I. Kuznetsov, Inhibitor protection of steel corrosion in acid solutions at high temperatures. A review. Part 2, Int. J. Corros. Scale Inhib., 2020, 9, no. 3, 867-902. doi: 10.17675/2305-6894-2020-9-3-5 
4. N.A. Gladkikh, M.A. Maleeva, L.B. Maksaeva, M.A. Petrunin, A.A. Rybkina, T.A. Yurasova, A.I. Marshakov and R.Kh. Zalavutdinov, Localized dissolution of carbon steel used for pipelines under constant cathodic polarization conditions. Initial stages of defect formation, Int. J. Corros. Scale Inhib., 2018, 7, no. 4, 683-696. doi: 10.17675/2305-6894-2018-7-4-14

5. S.M. Reshetnikov, T.A. Pisareva, E.V. Kharanzhevsky, F.Z. Gil'mutdinov and E.M. Borisova, Effect of laser treatment of unalloyed steel on the efficiency of benzotriazole as a corrosion inhibitor in a neutral medium, Int. J. Corros. Scale Inhib., 2020, 9, no. 3, 1113-1120. doi: 10.17675/2305-6894-2020-9-3-21

6. N.A. Gladkikh, Inhibition of local dissolution of metals by formulations based on organosilanes, Author's abstract of dissertation for the degree of Ph.D. (Chemical Sciences), speciality 05.17.03 - "Technology Electrochemical Processes and Corrosion Protection", Moscow, 2020, 16 pp. (in Russian).

7. V.I. Vigdorovich, L.E. Tsyganova, A.P. Pozdnyakov and N.V. Shel', Scientific basis, practice of creation and nomenclature of anticorrosive conservation materials. Tutorial, Tambov, Tambov State University, 2001, 192 pp. (in Russian).

8. I.N. Shabanova, L.V. Dobysheva, D.V. Varganov, V.G. Karpov, L.G. Kovner, O.I. Klyushnikov, Yu.G. Manakov, E.A. Makhonin, A.V. Khaidarov and V.A. Trapeznikov, New automated X-ray electron magnetic spectrometers: spectrometer with process attachments and manipulators, spectrometer for the study of melts, $I z \mathrm{v}$. $A N$ SSSR. Ser. Fiz., 1986, 50, no. 9, 1677-1682 (in Russian).

9. I.N. Shabanova, S.M. Reshetnikov, E.A. Naimushina and N.S. Terebova, XPS investigation of adsorption protective layers based on industrial inhibited oil, Int. J. Corros. Scale Inhib., 2020, 9, no. 3, 903-911. doi: 10.17675/2305-6894-2020-9-3-6 